\title{
Historia médica:
} Su potencialidad en la gestión de costos hospitalarios ${ }^{1}$

\section{Medical history: its potential in the management of hospital costs}

\author{
DOI: https://doi.org/10.17981/econcuc.40.1.2019.10
}

Artículo de Investigación. Fecha de recepción: 21/03/2019 Fecha de aceptación: 15/05/2019

Vilma Vargas González

Universidad del Atlántico. (Barranquilla, Colombia) vilmavargas@mail.uniatlantico.edu.co.

\section{Carmen Hernández Vargas}

Universidad del Zulia. (Barranquilla, Venezuela) cchiquinquira1990@gmail.com

Para citar este artículo:

Vargas, V. y Hernández, C. (2019). Historia médica: su potencialidad en la gestión de costos hospitalarios. Económicas CUC, 40(1). 157-170. DOI: http://doi.org/10.17981/econcuc.40.1.2019.10

\section{Resumen}

Desde el punto de vista de la información en salud, la historia médica juega un rol protagónico, como fuente de suministro de datos para la obtención de información para la gestión hospitalaria. Es por ello que este estudio pretende identificar las potencialidades de la historia médica, como fuente de suministro de datos necesarios para la obtención de información de costos, información de valiosa importancia para la gestión hospitalaria. En lo referente al diseño es una investigación de fuente mixta: Investigación de campo y documental, con alcance descriptivo. Se utilizó como técnica para la realización de la investigación la entrevista y como instrumento la guía de entrevista. Además, se realizó una revisión documental y articulación de teorías con evidencia empírica. Se encontró que la historia médica o expediente sanitario contiene, entre otros aspectos, la relación de los actos y procedimientos médicos realizados durante la estancia de un paciente en el centro hospitalario, actos y procedimientos médicos susceptibles de generar costos. Entre las potencialidades de la historia médica se encuentra que es una herramienta que permite el conocimiento de elementos de costos generados en la atención de los pacientes. En tal sentido, se concluye que la historia médica se vincula al sistema de información, siendo útil para la gestión hospitalaria y el control de los costos. Se concluye que la historia médica genera datos susceptibles de alimentar el sistema de información para la gestión de costos hospitalarios, en aras de suministrar servicios de calidad a los pacientes y optimización de la gestión.

Palabras clave: Historia médica; potencialidades; información de costos; gestión hospitalaria; control de costos

Clasificación JEL: I19, M41

\begin{abstract}
From the point of view of health information, medical history plays a leading role, as a source of data supply for obtaining information for hospital management. That is why this study aims to identify the potential of medical history, as a source of supply of necessary data to obtain cost information, information of valuable importance for hospital management. Regarding design, it is a mixed source investigation: field and documentary research, with descriptive scope. The interview was used as a technique for conducting the investigation and the interview guide as an instrument. In addition, a documentary review and articulation of theories with empirical evidence was carried out. It was found that the medical history or health record contains, among other aspects, the relationship of medical acts and procedures performed during a patient's stay in the hospital center, medical acts and procedures susceptible of generating costs. Among the potentialities of medical history is that it is a tool that allows the knowledge of cost elements generated in patient care. In this sense, it is concluded that the medical history is linked to the information system, being useful for hospital management and cost control. It is concluded that the medical history generates data that can feed the information system for the management of hospital costs, in order to provide quality services to patients and optimization of management.
\end{abstract}

Keywords: Medical history; potentials; cost information; hospital management; cost control.

\footnotetext{
${ }^{1}$ Artículo de investigación resultado parcial del proyecto de investigación Sistemas de información y gestión de costos en hospitales públicos de Barranquilla-Atlántico. Radicado N CE026-CDO2015 en Vicerrectoría de investigaciones, Extensión y Proyección Social de la Universidad del Atlántico (Colombia).
} 


\section{INTRODUCCIÓN}

La historia médica recopila cronológicamente todos los aspectos de la salud del paciente, y aquellos aspectos relevantes de la salud de su familia, que pudieran afectarlo directamente durante su vida; la historia médica o expediente sanitario, como también se le denomina, sirve de base para conocer las condiciones de salud del paciente, los actos médicos y procedimientos ejecutados para la recuperación de un estado patológico dentro de la relación médico-paciente.

Mediante la historia médica, se puede conocer los antecedentes de salud del paciente, los cuales permiten al médico tomar decisiones apropiadas en lo referente a la recuperación o conservación de un estado saludable, de acuerdo al tipo de medicina que se esté aplicando (curativa o preventiva). La recuperación o conservación de la salud de los pacientes, implica para la gestión hospitalaria, aplicación de recursos económicos, que a la postre pudieran traducirse en costos.

La gestión hospitalaria requiere de un sistema de información oportuno, que permita el conocimiento de los recursos aplicados en la atención de los pacientes; en tal sentido, se hace necesario instrumentos y herramientas que suministren datos confiables para alimentar el sistema. Con base en lo anterior, el objetivo del presente artículo es identificar las potencialidades de la historia médica, como herramienta fuente de suministro de datos necesarios, para la obtención de información de costos.

El conocimiento de costos es trascendental para la gestión hospitalaria, en tanto información útil en la toma de decisiones para la prestación de servicios de calidad bajo parámetros de eficiencia y eficacia en consonancia con la preparación de presupuestos para un manejo adecuado de los recursos disponibles.

\section{Metodología}

La metodología utilizada tiene una orientación cualitativa. En lo referente al diseño es una investigación de fuente mixta: Investigación de campo y documental, con alcance descriptivo. Se utilizó como técnica para la realización de la investigación la entrevista y cómo instrumento la guía de entrevista. Además se realizó revisión documental y articulación de teoría con evidencia empírica, y discusión grupal a partir del análisis de teorías sobre sistemas de información. Se consideran los postulados teóricos sobre sistemas de información, gestión y control de costos de: Anthony y Govindarajan (2008); López (2007); Horngren, Datar y Rajan (2012); Oz (2008); Polimeni, Fabozzi y Adelberg (1994) y Senn (1999).

Para el logro del objetivo planteado se seleccionaron hospitales representativos de la red hospitalaria de la ciudad de Barranquilla, por su nivel de complejidad y cobertura poblacional. Se realizó entrevistas a personal clave de dichas instituciones hospitalarias, y revisión documental, entre ellos los formatos de historia médica general (sin diligenciar), utilizados en los hospitales objeto de estudio; en tal sentido, se efectuó una revisión de los datos solicitados en dichos formatos, los cuales deben ser diligenciados por el personal médico tratante; además se realizó un esfuerzo de articulación de la teoría con evidencia empírica.

En aras de claridad en el discurso, se realizan las siguientes consideraciones: 
Primero: "el valor en dinero que se da a un insumo se calcula multiplicando una cantidad física por un precio unitario. El resultado es una suma monetaria que se llama costo; El costo es una medida monetaria del monto de los recursos usados por un centro de responsabilidad" (Anthony y y Govindarajan, 2008, p. 130), de acuerdo con los autores antes citados, los pacientes de un hospital no son insumos, más bien, los insumos son los recursos que usa el hospital para cumplir el objetivo de tratar a los pacientes, de esta forma consideran, es más fácil medir el costo de los insumos que calcular el valor de los productos. En tal sentido, son elementos de costo de los productos de acuerdo con Polimeni, et al. (1994), los materiales directos, la mano de obra directa y los costos indirectos de fabricación o de prestación del servicio. Esta clasificación suministra información necesaria para la gestión y el control, tal como medición del ingreso, fijación de precios del producto o del servicio y elaboración de presupuestos.

Segundo: La Real Academia de la Lengua Española (RAE, 2018), define fuente (en una de sus acepciones), como: una persona o cosa que proporciona información. $\mathrm{Y}$ fuentes bibliográficas o documentales se definen como el origen de información utilizada en la realización de investigaciones, estas fuentes contienen datos formales, informales, escritos, u orales. Para Silvestrini y Vargas (2008) las fuentes bibliográficas o documentales, se dividen en tres tipos: primarias, secundarias $y$ terciarias, donde, las fuentes primarias contienen información original, que ha sido publicada por primera vez y que no ha sido filtrada, interpretada o evaluada por nadie más. Pueden encontrarse en formato tradicional impreso como los libros, las publicaciones seriadas o en formatos especiales.
Por otra parte, la información de costos generada a partir de los datos suministrados por la historia médica, puede incidir sobre la oferta de servicios porque, al conocer la rentabilidad, la utilidad o la pérdida ocasionada por un procedimiento en una institución hospitalaria, se puede tomar la decisión de eliminarlo, estimularlo o mantenerlo, aunque no genere ganancias, pero proporcione beneficios a la comunidad (Idárraga, Acevedo y Ramírez, 2001).

En tal sentido, en el siguiente aparte, se presenta un referente conceptual sobre la clasificación de los costos, con el propósito de facilitar la comprensión de la utilidad de la información de costos que puede ofrecer la historia médica.

\section{Fundamentación TeóRICA}

En este aparte se realiza un esfuerzo de clasificación de los costos, tomando en consideración los postulados teóricos de Horngren, et al.,(2012); Matz y Usry (1980); Polimeni et al. (1994); Welsch (1979). En tal sentido, la tabla 1 muestra los costos clasificados en relación a distintas variables, tales como: volumen de actividad, producción, identificación con el producto o servicio, funciones, tiempo de cálculo, control, y toma de decisiones. Se hace hincapié en considerar a la clasificación anterior como no definitiva ni única, pero sí de uso frecuente; por tanto se entiende que existen otras, y no hay líneas divisorias tajantes entre unas y otras; en tal sentido, es bueno aclarar que las particularidades de cada institución propician la determinación de su clasificación de costos.

En este aparte se revisa la clasificación relacionada con el volumen de actividad, identificación con el producto o servicio, control y toma de decisiones, por considerarlas apropiadas al desarrollo de la temática. 


\section{Volumen}

En relación al volumen de actividad, los costos se clasifican en fijos, variables y mixtos:

\section{Costos fijos}

Son aquellos que no varían con la producción o la actividad productiva y se acumulan con el transcurso del tiempo, permaneciendo constantes en valor durante periodos a corto plazo, dentro de un rango relevante de actividad. El rango o trayecto relevante de actividad es el intervalo de actividad (recorrido de actividad) dentro del cual los costos fijos totales y costos variables unitarios (tasa de variabilidad) permanecen sin cambios o constantes. De acuerdo con Polimeni et al. (1994) "son aquellos cuyo costo fijo total permanece constante dentro de un rango relevante de producción, mientras el costo fijo por unidad varía con la producción, más allá del rango relevante de producción variarán los costos fijos" (p. 15). El responsable de estos costos es la gerencia, quien controla el volumen de producción.
Los costos fijos son ocasionados por el mantenimiento de activos y otros factores de producción en un estado de disposición a producir o prestar servicios, esto hace que se denominen costos de capacidad. Dada la relación entre capacidad y costos fijos, al aumentarse la capacidad instalada se incrementa los costos fijos, sin embargo, no siempre que hay un incremento de costos fijos, esto implica un aumento de capacidad. Los costos fijos responden principalmente a dos tipos: un primer tipo corresponde a aquellos costos establecidos por disposiciones administrativas previas, no se tiene poder sobre ellos para suspender su incurrencia. Este tipo de costos se les denomina frecuentemente como costos fijos comprometidos, "sumergidos" o "muertos", tal es el caso, por ejemplo, de los costos por depreciación de equipos médico-quirúrgicos, impuestos, o seguros.

Un segundo tipo de costos fijos corresponde a aquellos establecidos por decisiones administrativas en una base a corto plazo, y susceptibles de ser modificados, pueden llegar a fluctuar por razón de cambio en

TABLA 1

Clasificación de costos

\begin{tabular}{|c|c|}
\hline Relación a $\downarrow$ & se clasifican en $\downarrow$ \\
\hline \multirow{2}{*}{ Volumen } & Mixtos \\
\hline & Semivariables y escalonados \\
\hline Producción & Conversión* \\
\hline $\begin{array}{l}\text { Identificación con una Actividad, } \\
\text { Departamento, Producto o servicio }\end{array}$ & Directos e indirectos \\
\hline Funciones (áreas funcionales) & Producción, mercadeo, administrativos y financieros ${ }^{*}$ \\
\hline Tiempo en que se calculan & Históricos y predeterminados* \\
\hline Período en que se comparan con los ingresos & Producto y periodo* \\
\hline Control & Controlables y no controlables \\
\hline Toma de decisiones & Diferenciales y de oportunidad \\
\hline
\end{tabular}


la estructura básica de la empresa, si se suspende una determinada actividad en la empresa o por cambios discrecionales en la política administrativa; en este sentido, a este tipo de costos se les denomina costos fijos discrecionales, tal es el caso de sueldos y publicidad.

De acuerdo con (Welsch, 1979) los principales factores que una institución debe considerar al establecer una definición de costos fijos, son los siguientes: Controlabilidad; relación con la actividad; rango pertinente o relevante de actividad; regulados por la administración; costos por tiempo; fijos en total pero variables por unidad. En este aspecto, un costo fijo es constante en valor total a cada periodo; sin embargo, cuando se ve en términos de unidades de producción, tiene un efecto variable sobre el costo unitario. El costo total permanece constante independientemente de la cantidad producida, mientras que el costo unitario varía inversamente con respecto al volumen, es decir, a mayor volumen menor costo variable y viceversa; Aplicación práctica, en el sentido que, consideraciones prácticas no exigen que un costo sea absolutamente fijo. En la aplicación, un costo fijo es uno que es constante para los fines prácticos. Implica para la gestión de costos, en el supuesto que los demás factores permanezcan constantes, que la actividad productiva o prestación de servicios deba expandirse hasta donde la capacidad instalada lo permita, lo cual reducirá el costo fijo unitario.

\section{Costos variables}

En relación a los costos variables, estos pueden ser definidos como "aquellos en los que el costo total cambia en proporción directa a los cambios en el volumen, o producción, dentro del rango relevante, en tanto que el costo unitario permanece constante" (Polimeni et al., 1994, p. 15); de forma tal que si los costos variables cambian en proporción directa con los niveles de actividad, se puede decir que son costos de actividad porque se acumulan como resultado de la producción, volumen de la actividad o del trabajo realizado en la prestación de servicios y no existirían si no fuera por la realización de una actividad (a cero actividad, cero costo variable). Los costos variables totales aumentan o disminuyen en proporción directa con los cambios en la producción como patrón de comportamiento.

Según (Welsch, 1979), los factores principales a tomar en consideración para definir costos variables son: controlabilidad; relaciones proporcionalmente con la actividad; rango pertinente o relevante; regulados por la administración; costos de actividad; variables en total pero fijos por unidad. Un costo variable lo es cuando se relaciona con la producción total, sin embargo, es constante si se mira como costo unitario. Como consideraciones prácticas se tiene que un costo variable no tiene que ser absolutamente variable en su aplicación y entre las implicaciones para la gestión de estos costos en el supuesto de que los demás factores se mantuvieran constantes, de acuerdo con Polimeni et al. (1994) cada expansión deseada de actividad productiva por unidad ocasionaría un cambio incremental en los costos variables totales igual a un monto constante por unidad. En la medida en que el precio de venta por unidad exceda el costo variable por unidad debe expandirse la actividad productiva.

\section{Costos mixtos}

Por su parte, los costos mixtos son aquellos que poseen características tanto de los costos fijos como de los variables a lo largo de varios rangos pertinentes de operación. 
De acuerdo a lo expresado por Matz y Usry (1980) existen tres razones para clasificarlos de esta forma:

1. La necesidad de tener una organización mínima, o de consumir una cantidad mínima de accesorios o de servicios a fin de mantenerse listos para operar. Más allá de este mínimo, que es fijo, los costos adicionales varían con el volumen.

2. Las clasificaciones contables basadas en el objeto de la erogación o función por lo general agrupan juntas las partidas fijas y variables. Como ejemplo, el costo de electricidad puede cargarse a una sola cuenta a pesar de que el costo de electricidad usado para climatizar las oficinas administrativas tiene un consumo en kilovatios hora diferente a la cantidad de kilovatios hora consumidos en los departamentos productivos, el cual dependerá del volumen de producción o prestación del servicio.

3. Los factores de producción son divisibles en unidades infinitamente pequeñas. Cuando tales costos son comparados en un cuadro contra sus volúmenes, sus movimientos aparecen como una serie de pasos, más bien que como una línea recta continua. Esta situación es completamente perceptible al cambiarse una operación de un solo turno a dos turnos, o de dos turnos a tres turnos. Tal movimiento resulta en pasos bien definidos en la línea de costos debido a que una serie de costos, debe agregarse a un punto dado (p. 625)

Los costos mixtos, además se subclasifican en semivariables y escalonados:

\section{- Costos semivariables}

Son partidas de costos que aumentan o disminuyen según disminuye o aumenta el volumen de actividad, pero no en proporción directa, lo cual implica que estos costos despliegan, como hemos mencionado en líneas anteriores, características de costos fijos y variables; "la parte fija usualmente representa un cargo mínimo al hacer determinado artículo o usar un servicio.
La parte variable es el costo cargado por usar realmente el servicio" (Polimeni et al., 1994, p. 19).

La variabilidad de los costos semivariables se atribuye al efecto combinado del transcurso del tiempo, la actividad de producción o prestación de servicios y las decisiones discrecionales de política administrativa. La primera y segunda razones, expuestas por Matz y Usry (1980) para clasificar los costos en mixtos atienden a la subclasificación de costos semivariables. Entre estos costos se encuentran los costos de servicios públicos, tales como electricidad, telefóno, etc.

\section{- Costos escalonados}

La parte fija de los costos escalonados cambia abruptamente a diferentes niveles de actividad puesto que se adquieren en partes indivisibles. Un costo escalonado es similar a un costo fijo dentro de un rango relevante muy pequeño (Polimeni et al., 1994). La tercera razón expuesta por Matz y Usry (1980) para clasificar los costos en mixtos, atiende a la subclasificación de costos escalonados y es el caso, por ejemplo, del cambio de un turno a dos turnos en un centro hospitalario, donde una serie completa de enfermeras o médicos de guardia, etc, deben agregarse en un punto dado.

\section{Producción}

En relación a la producción o prestación de servicios, los costos se clasifican en costos primos y costos de conversión.

\section{Costos primos}

Esta clasificación está dirigida a aquellos relacionados en forma directa con producción o prestación de servicios, como por ejemplo: mano de obra y materiales directos. 


\section{Costos de conversión}

Son aquellos costos relacionados con la transformación de los materiales directos en productos terminados, se consideran en esta clasificación, por ejemplo: la mano de obra directa y los costos indirectos de fabricación. Tomando en consideración la clasificación, encontramos que los costos considerando la capacidad de la gerencia para identificarlos o asociarlos con una actividad, departamento, producto o servicio se pueden clasificar en costos directos e indirectos, así:

\section{- Costos directos}

Pueden asociarse directamente a un producto o a una unidad organizativa. Esta clasificación tiene sentido cuando se identifica la unidad de referencia. Los materiales directos y los costos de mano de obra directa de un determinado producto son ejemplo de costos directos.

\section{- Costos indirectos}

Son los gestados entre varias unidades de referencia, bien porque resulte excesivamente oneroso identificar su relación con una unidad, bien porque sean costos de recursos compartidos entre varias. A diferencia de los directos los costos indirectos han de ser repartidos entre las unidades que los generan (Pérez-Carballo, 2006). Usualmente los costos indirectos se cargan a los artículos o áreas (departamentos, centros de costos, etc), con base en técnicas de asignación, las cuales involucran la distribución de los costos indirectos de producción o prestación del servicio entre las diversas categorías, mediante una tasa de aplicación, esta tasa de aplicación se calcula dividendo los costos indirectos entre la capacidad productiva o de prestación del servicio, tanto el numerador como el denominador generalmente son estimados; el denominador se fija mediante alguna base, denominada actividad del denominador, que debe estar relacionada directamente con los costos indirectos de producción estimados usados en el numerador para el cálculo de la tasa.

Toma de decisiones

En relación a la toma de decisiones los costos se clasifican en:

\section{Costos Diferenciales}

Están constituidos por la diferencia entre los aumentos o disminuciones en el costo total, o el cambio en cualquier elemento de costo, generado por cursos alternativos de acción. Si el costo aumenta de una alternativa a otra se denomina costo incremental; si el costo disminuye de una alternativa a otra, se denomina costo decremental. Estos costos son importantes en el proceso de toma de decisiones, pues son quienes muestran los cambios o movimientos sufridos en las utilidades de la empresa (Backer, Lyle y Padilla, 1988; Polimeni et al., 1994). Al realizar el análisis de una decisión la clave de dicho análisis se encuentra en el estudio de los efectos de la diferencia de costos que genera cada opción en las utilidades.

\section{Costos de oportunidad}

Cuando se toma una decisión para empeñarse en determinada alternativa, se abandonan los beneficios de otras opciones. Los beneficios perdidos al descartar la siguiente mejor alternativa son los costos de oportunidad de la acción escogida. 
Los costos de oportunidad no se incluyen en los registros contables, sin embargo, para propósito de toma de decisiones deben tomarse en consideración para hacer las evaluaciones necesarias a las alternativas propuestas (Backer,et al., 1988, Polimeni et al., 1994; Horngren et al., 2012).

Control

En relación al Control, los costos se clasifican en:

\section{Costos Controlables y no Controlables}

Son aquellos sobre los cuales la decisión de una persona, a determinado nivel, tiene autoridad suficiente para su incurrencia o no. Están directamente influenciados por el gerente de un departamento o centro de costos en un periodo dado. Los no controlables son aquellos costos en los que el gerente de la unidad no tiene autoridad sobre su incurrencia.

En ese orden de ideas, el control de costos, aspecto fundamental en la gestión hospitalaria, requiere de fuentes de suministro de información para su efectiva realización.

\section{Resultados}

En relación a las anteriores consideraciones, en la siguiente sección, se presentan los resultados de la investigación. Comenzando con las características de la historia médica que la convierten en herramienta fuente de datos para la gestión hospitalaria y el control de costos.

Características y potencialidades de la Historia Médica en la gestión de costos hospitalarios

Un conocimiento sólido de las características de los costos es fundamental para que los administradores observen la necesidad de su uso racional. Este amplio conocimiento permite clasificarlos y priorizarlos atendiendo al tipo de decisión que se esté tomando y la identificación de la relación que existe entre la capacidad instalada y su correspondiente estructura de costos.

Cuando se trata de información referente a los actos y procedimientos ejecutados para recuperar al paciente dentro del centro hospitalario, -aspecto propio de la medicina curativa-. La información contenida en la historia médica nos permite conocer elementos de costos referidos a los recursos empleados para restablecer al paciente desde el momento de su ingreso (admisión) hasta su egreso ya sea por alta médica (curación o mejoría), contra opinión médica, contrareferencia o por defunción.

La historia médica, por ser una herramienta asistencial todavía insustituible, presenta problemas para su incorporación en el seno de un sistema de información, principalmente cuando se aspira su automatización al intentar establecer modelos que contengan el gran volumen de datos de la historia médica, que permitan un rápido acceso a su contenido por medios informáticos y faciliten la consulta retrospectiva de los episodios asistenciales de un paciente, así como el tratamiento estadístico de los datos que contiene, con objetivos principalmente no asistenciales sino de investigación, epidemiológicos, gestión, planificación y control de los servicios de salud (López, 2007).

Esta herramienta asistencial tiene una serie de características, las cuales se señalan a continuación, por su valor para la temática y el discurso desarrollado, en lo atinente a los sistemas de información para la gestión hospitalaria y el control de costos. 
a. Acceso y Confidencialidad. La discusión acerca de la propiedad de la historia médica, aún vigente, conduce a un problema de carácter ético sobre la discrecionalidad de su uso y el acceso a la misma. "La historia clínica es propiedad de los pacientes, el servicio de salud es el depositario de ella con el fin de proporcionársela a aquellos profesionales que la necesiten para el desempeño de sus funciones" (Cáceres, García, Alejo y Pijierro, 2007, p. 161), lo cual supone una dificultad al sistema de información en tanto, el acceso a la información contenida en ella, no puede ser usada por personal ajeno a los profesionales de la salud en desempeño de funciones para restablecer la salud del paciente. Sin embargo, esta supuesta dificultad no es obstáculo para incorporar al sistema la información contenida en ella, al ser una cuestión de organizar el sistema de tal manera que el acceso a cierto tipo de información confidencial solo pueda ser vista por personal autorizado, para mantener la seguridad de los datos, la confidencialidad y la autenticidad de la historia médica.

b. Obligatoriedad de realización. "La obligatoriedad de llevar o registrar los acontecimientos de salud por parte de los profesionales y las instituciones, que para ello intervienen, está dado en forma explícita por algunas legislaciones, como las de Colombia, España, Perú, Argentina, Estados Unidos, Reino Unido y Francia" (Rueda-Clausen, 2006, p. 64), En la práctica, las instituciones prestadoras de servicios de salud registran los acontecimientos de salud de los pacientes que acuden a ellas, siguiendo un formato preestablecido, ya sea manual o informatizado, de acuerdo a las tecnologías de información y comunicación (TIC). Puede generar información administrativa, técnica, de carácter probatorio y epidemiológico. c. Base para información epidemiológica. La historia clínica es básica para obtener información sobre Enfermedades de Declaración Obligatoria (EDO). Distintas legislaciones establecen la obligatoriedad de declarar cierto tipo de enfermedades que por sus características de riesgo para la población pudieran degenerar en epidemias o problemas de salud de alto riesgo para la población, a fin de tomar las medidas preventivas a nivel de salud pública, y que los organismos competentes ejerzan la vigilancia epidemiológica a que haya lugar, En este sentido, enfermedades tales como el SIDA y las enfermedades infectocontagiosas de rápida propagación, entre otras, son de obligatoria declaración, sin embargo, es necesario acotar con "toda la información que concierne a un paciente debe manejarse de acuerdo con principios deontológicos. En las aplicaciones estadísticas, la información sobre los pacientes puede tratarse siempre que se respete la confidencialidad" (Gil-Redrado, 2007, p. 133).

d. Instrumento de análisis técnico. El registro del historial médico conlleva aspectos de diferente naturaleza, entre estos cabe destacar el ser un documento jurídico de carácter probatorio y por ende sujeto a los requerimientos de la Ley. Contiene un análisis de los procedimientos practicados a un paciente y con estos registros se puede medir la calidad de la prestación del servicio; en este sentido es un documento de carácter técnico y puede ser objeto de estudio. Adicional, en la medida de lo posible, la historia médica es única, integral, secuencial, clara, oportuna $\mathrm{y}$ comprensible.

Las anteriores características implican para la gestión hospitalaria, el mantenimiento de una capacidad instalada; la cual genera costos a la institución. Por tanto, es pertinente una discusión que sirva de guía en la clasificación de los costos para el control y gestión hospitalaria. 
Información de costos suministrada por la historia médica

El Ministerio de salud y protección social de Colombia en el año 2012 puso a disposición de los usuarios el Sistema Único de Información en Salud (Ospina, 2012). Según lo expresado, entre los beneficios de contar con un Sistema Único de Información en salud se encuentran:

La estandarización de los flujos de información, evitando la duplicidad de requerimientos por parte de las entidades territoriales $\mathrm{u}$ organismos de control. Se optimizará el uso de la información existente en el país sobre salud para una mejor toma de decisiones y con la exposición de dicha información, esta vaya mejorando su calidad desde la fuente primaria (Ospina, 2012).

Dado lo anterior, es pertinente acotar que en la historia médica se realiza el registro de los medicamentos y dosis aplicadas al paciente, cantidad de servicios de diagnóstico utilizados, tiempo utilizado por él o los médicos en la revisión y atención al paciente, especialidad del o los médicos tratantes, procedimientos quirúrgicos, cantidad de material médico-quirúrgico utilizado, estancia hospitalaria del paciente en la institución, entre otros aspectos, que son susceptibles de convertirse en información administrativa- contable, de manera que, la historia médica es un documento clave en el conocimiento de datos relacionados con elementos de costos.

En un hospital, los costos fijos, están dados por la capacidad instalada para la efectiva producción de un bien o servicio; por ejemplo, la cama hospitalaria aun cuando permanezca vacía, implica costos en los centros hospitalarios. Por el contrario, los costos variables dependen de los niveles de prestación del servicio. La relación entre producción y costos fijos constituye un importante indicador de eficiencia, que representa el nivel de aprovechamiento de la capacidad hospitalaria instalada, y se puede analizar mediante indicadores tales como: día cama ocupada o paciente -día (Indicador de Costo Variable) y el día-cama (Indicador de Costo Fijo).

El día cama ocupada contiene el costo del conjunto de actividades realizadas para recuperar la salud del paciente que se encuentra hospitalizado; por día-cama ocupada debe entenderse "cada período de 24 horas durante los cuales una cama de hospitalización permanece ocupada por un paciente" (García, 1993, p. 114). El día cama ocupada incluye costos variables directos e indirectos, además del costo de los cuidados médicos directos que recibe el paciente en el servicio hospitalario, los otros servicios de apoyo para su atención médica integral, relacionados con diagnóstico, tratamiento, recuperación y rehabilitación y por supuesto todos los servicios de hotelería que consume el paciente durante el tiempo que permanece hospitalizado. En cambio el costo del día-cama expresa las sumas pagadas por personal, tecnología, planta física, mantenimiento, etc, disponibles para atender la demanda de los pacientes.

Según Vivas (2018) en el Registro Especial de Prestadores de Servicios de Salud (REPS), Colombia cuenta con 84.556 camas hospitalarias para aproximadamente 49 millones de habitantes y aunque en la Costa Caribe la tasa promedio de camas instaladas por cada mil habitantes casi dobla la media nacional, la presencia de entidades estatales no supera el $30 \%$.

La cama instalada y la cama ocupada generan costos a las instituciones prestadoras de servicios de salud. El día cama ocupada y la estancia hospitalaria son indicadores susceptibles de ser verificados en los registros estadísticos de los centros hospitalarios con soporte en la historia médica. 
La estancia del paciente en el centro hospitalario queda registrada en la historia médica, dato que es utilizado por el departamento de registros y estadísticas de salud de los hospitales para calcular el promedio de estancia, información de fundamental importancia para los costos. El promedio de estancia, indica los días promedio de permanencia de los pacientes que egresaron (altas hospitalarias) en el periodo en el hospital.

El cálculo del promedio de estancia sirve para evaluar "prolongación innecesaria de los días de hospitalización, incoordinación entre los servicios administrativos, auxiliares, diagnóstico o terapéuticos y los servicios de hospitalización" (García, 1993, p. 117). En tal sentido, la estancia hospitalaria, estaría condicionada a procedimientos tanto administrativos, como técnicos.De acuerdo con Vargas y Hernández (2007):

Las prolongaciones innecesarias ya sea por causa administrativa o científica (deficiencias en la calidad técnica o prestación inapropiada de los servicios), genera costos por hospedaje y terapéuticos a la institución hospitalaria, que se traducen en ineficiencia y poca productividad (p. 450).

Existen diversos métodos validados que se utilizan para la evaluación de la adecuación de la admisión del paciente y la estancia; entre ellos destaca el Appropriateness Evaluatión Protocol (AEP), su aplicación demuestra que existen cifras relativamente altas de ingresos inapropiados (41-56\% en hospitales americanos o italianos) o estancias inapropiadas (hasta 54\%). En España aplicando criterios semejantes, se han encontrado ocasionalmente en un pasado no lejano tasas de estancias inapropiadas de hasta el $60 \%$ (Del Rio, Terol, Cortes, Dotor y Temes, 2007, p. 84).
Hospitales de la ciudad de Barranquilla, mantuvieron para el periodo 2006-2010, un promedio de estancia de 4,$5 ; 3,3 ; 3,6$; 2 y 1,8 días respectivamente para cada año del periodo (Alcaldía de Barranquilla, 2011). En cualquier situación, el patrón de comparación debe ser establecido por cada institución hospitalaria tomando en consideración su nivel de complejidad o mejor la complejidad de las patologías que atiende y sus especialidades y así poder hacer uso del AEP.

El promedio de estancia o estancia media depende del tipo de pacientes que se traten en la unidad o servicio hospitalario, es decir del tipo de complejidad manejada en el servicio o tratamiento que necesite el paciente. Lo cual implica que al comparar el promedio de estancia de diversas instituciones hospitalarias, las diferencias pueden provenir tanto de una mayor o menor eficiencia de las instituciones, como de los diferentes casos o patologías atendidas, este último aspecto justificaría una mayor duración de la estancia. Una alternativa es ajustar el indicador por tipo de pacientes atendidos en cada institución, mediante algún sistema de clasificación de pacientes, como los grupos de diagnósticos relacionados -GDR(Vargas y Hernández, 2007).

Para tener un conocimiento aproximado de donde provienen las diferencias en estancia media entre hospitales (Peiró, 1999), propone el cálculo de la estancia media ajustada por funcionamiento, esto es, la estancia media que tendría un hospital con los pacientes por grupo de diagnóstico relacionado con los atendidos, con lo cual se ofrece una idea de la complejidad del hospital. También propone el cálculo de la estancia media ajustada por casuística, aquella que tendría un hospital si tratara la misma casuística que el 
patrón de referencia, pero con sus estancias medias en cada grupo de diagnóstico relacionado, con el cual se controla la casuística como factor de confusión en la prolongación de la estancia media.

Tomando en consideración estos planteamientos, en un hospital con bajo nivel de información cuantitativa, los juicios se basan en las percepciones y las opiniones de los individuos presuntamente más preparados para emitirlos. Muchas de las opiniones extendidas sobre los servicios hospitalarios no tienen otra base que el sentido común de los profesionales del sector, y, en algunos casos, al disponer de información objetiva no se sostienen (Casas, 2007), aun cuando, de acuerdo con Testa (1995) "no se puede admitir en la misma forma el supuesto de utilidad válido para la actividad específicamente económica" (p. 10). Como ya hemos mencionado, implica que el cálculo de costos no tiene como objetivo la búsqueda de la rentabilidad económica, sino la optimización del uso de los recursos desde el punto de vista social.

Es decir, el desarrollo de sistemas de costos para hospitales públicos no debería estar determinado por el cálculo de utilidad en el sentido económico del término, sino en función del beneficio social que el conocimiento de información de costos generaría para un mejor uso de los recursos, en beneficio del mayor número de usuarios atendidos. Para esto se requiere en primer lugar el registro de cada uno de los elementos de costo necesarios para la prestación del servicio: -insumos, bienes de capital y personal-; pero además el cálculo de costos debe incluir de acuerdo con Testa (1995): "la lógica de los instrumentos y de las actividades y la lógica de la organización de los servicios de salud" (p. 126).

\section{Conclusiones}

La historia médica, es una herramienta de uso significativo en la atención de los pacientes, en tanto suministra información relacionada con los procedimientos e intervenciones médicas a las cuales son sometidos en el transcurso de su estadía en el hospital; misma información revela datos relacionados con los elementos de costos necesarios en la atención del paciente a fin de recuperar su estado de salud. En tal sentido, además se concluye, como la historia médica genera datos susceptibles de alimentar el sistema de información para la gestión de costos hospitalarios, en aras de suministrar servicios de calidad a los pacientes y optimización de la gestión.

La clasificación de costos de acuerdo a las especificidades y necesidades de las instituciones, posibilita a los administradores y gerentes médicos, bases sólidas sobre la cantidad de recursos necesarios en el proceso de prestación de los servicios hospitalarios y toma de decisiones sobre su oferta.

Las instituciones hospitalarias poseen la capacidad administrativa y las herramientas para el desarrollo de sistemas de costos que permitan suministrar información necesaria para la toma de decisiones gerenciales y ahí radica la importancia de la historia médica como el documento que proporciona a la gestión hospitalaria, datos fundamentales para la construcción de estructuras de costos de los servicios hospitalarios.

El conocimiento de la estructura de costos aporta a la gestión hospitalaria el control de información que puede incidir en los presupuestos, la oferta de servicios y uso de los recursos; y en ese sentido, se infiere sobre la importancia y potencialidad de la historia médica para la gestión y control de costos hospitalarios. 


\section{REFERENCIAS}

Alcaldía de Barranquilla. (2011). Mantenimiento en la producción de servicios hospitalarios con menores estancias, menos hospitalizados, más cirugias. Recuperado de www.barranquilla.gov.co/normas/Indicadores_2.ppt

Anthony, R. y Govindarajan, V. (2008). Sistemas de control de gestión. (12 ed). México, D.F.: Mac Graw Hill.

Asociación de academias de la lengua española. (2014). $R A E$. Recuperado de https://dle.rae.es/?id=IYZhVtl

Backer, J., Lyle, J. y Padilla, D. (1988). Contabilidad de Costos. Un enfoque administrativo para la toma de decisiones. (2 ed.). México, D.F.: McGrawHill.

Cáceres, F., García, F., Alejo, J. y Pijierro, A. (2007). El sistema integrado de información sanitaria en un servicio de salud. (4 ed.). Madrid: McGraw-Hill Interamericana.

Casas, M. (2007). La información para la gestión clínica. En, J. Temes y M. Mengíbar (comp.), Gestión Hospitalaria. (4 ed.). Madrid: McGraw-Hill Interamerican.

Del Rio, U., Terol, J., Cortes, C., Dotor, M. y Temes, J. (2007). Procesos asistenciales integrados. En, J. Temes y M. Mengíbar (comp.), Gestión Hospitalaria. (4 ed.). Madrid: McGraw-Hill Interamerican.

Gil-Redrado, J. (2007). Indicadores Sanitarios. En, J. Temes y M. Mengíbar (comp.), Gestión Hospitalaria. (4 ed.). Madrid: McGraw-Hill Interamerican.

García, J. (1993). Indicadores de Gestión para Establecimientos de Atención Médica. Guía Práctica. Caracas: Disinlimed.

Horngren, C., Datar, S. y Rajan, M. (2012). Contabilidad de costos. Un enfoque gerencial. (14 ed.) México, D.F.: Pearson Educación.
Idárraga, M., Acevedo, M. y Ramírez, R. (2001). Gerencia de los Procesos Administrativos. En, J. Toro, Hospital y Empresa. Medellín: Hospital Universitario San Vicente de Paúl.

López, O. (2007). Los servicios de admisión $y$ documentación clínica. (4 ed.). Madrid: McGraw-Hill Interamericana.

Matz, A. y Usry, M. (1980). Contabilidad de Costos. Planificación y control. Cincinnati: South-Western Publishing.

Ospina, M. (noviembre 26, 2012). Sistema único de información en salud, al servicio del país. [Boletín de prensa $\mathrm{N}^{\circ}$ 389]. Ministerio de salud y protección social. Recuperado de https://www. minsalud.gov.co/Paginas/Sistema-unico-de-Informaci\%C3\%B3n-en-Saludal-servicio-del-pa\%C3\%ADs.aspx

Oz, E. (2008). Administración de los sistemas de información. (5 ed.) México, D.F.: Thomson.

Polimeni, R., Fabozzi, F. y Adelberg, A. (1994). Contabilidad de Costos. Conceptos y aplicaciones para la toma de decisiones gerenciales. Bogotá, D.C.: McGraw.

Pérez-Carballo, J. (2006). Control de la Gestión Empresarial. Textos y casos. (8 ed.). Madrid: Esic editorial

Peiró, S. (1999). Medidas de actividad y producto sanitario. En, J. Llano (ed.), Gestión sanitaria. Innovaciones y desafíos. Barcelona: Massón.

Rueda-Clausen, C. (2006). La historia clínica informatizada. Evaluación de los casos colombiano y español. MedUNAB 9(1).63-71. Recuperado de http:// caribdis.unab.edu.co/pls/portal/url

Senn, J. (1999). Análisis y diseño de sistemas de información. (2 ed.) México, D.F.: McGraw-Hill.

Silvestrini, M. y Vargas, J. (2008). Fuentes de información primarias, secundarias y terciarias. [Presentación]. Recuperado de http://ponce.inter.edu/cai/ manuales/FUENTES-PRIMARIA. pdf 
Testa, M. (1995). Pensamiento estratégico y lógica de programación. (El caso de la salud). Buenos Aires: Lugar.

Vargas, V. y Hernández, E. (2007). Indicadores de Gestión Hospitalaria. Revista de Ciencias Sociales, 13(3). 444-454.

Vargas, V. (2010). Sistemas de información de costos en salud. [Trabajo para ascenso]. Universidad del Zulia, Venezuela.

Vivas, J. (julio 30, 2018). Colombia cuenta con 1.7 camas hospitalarias por cada 1000 habitantes. El Tiempo. [Otras ciudades]. Recuperado de https:// www.eltiempo.com/colombia/otrasciudades/colombia-solo-cuenta-con1-7-camas-hospitalarias-por-cadamil-habitantes-249374.

Welsch, G. (1979). Presupuestos: planificación y control de utilidades. México, D.F.: Prentice-Hall Hispanoamericana.

\section{Biodata}

Vilma Vargas González es Contador Público. Magíster en Gerencia Pública. Doctora en Ciencias Sociales- Mención Gerencia por la Universidad del Zulia, Venezuela. Docente del programa de Contaduría Pública de la Universidad del Atlántico, Investigadora adscrita al grupo de investigación Organizaciones sostenibles de la Universidad del Atlántico, acreditado por Colciencias. https://orcid.org/0000-00027796-0099

Carmen Hernández Vargas es Médico cirujano, Estudiante del tercer año del Programa Especialización en Medicina Interna del Postgrado de Medicina de la Universidad del Zulia, Investigadora acreditada ante el Fondo Nacional de Investigaciones Científicas y Tecnológicas (FONACIT), Venezuela. https://orcid.org/0000-0001-56612346 